\title{
Colonic Ulcer, CTCAE
}

National Cancer Institute

\section{Source}

National Cancer Institute. Colonic Ulcer, CT CAE. NCI Thesaurus. Code C143378.

A disorder characterized by a circumscribed, erosive lesion on the mucosal surface of the colon. 